\title{
Comparison of Clonidine and Fentanyl Premedication on Hemodynamic Factors in Opium-Dependent Patients Under Endotracheal Intubation
}

\author{
Hamzeyi A. ${ }^{1}$ MD, Basiri Moghaddam M. ${ }^{2}$ MSc, Mohammadpour A. ${ }^{3}$ PhD, Talayi A.R.* $M S c$
}

\begin{abstract}
*Nursing Department, Nursing \& Midwifery Faculty, Gonabad University of Medical Sciences, Gonabad, Iran ${ }^{1}$ Anesthesia \& Operating Room Department, Paramedical Faculty, Gonabad University of Medical Sciences, Gonabad, Iran

${ }^{2}$ Nursing Department, Nursing \& Midwifery Faculty, Gonabad University of Medical Sciences, Gonabad, Iran Internal Surgery Nursing Department, Nursing \& Midwifery Faculty, Gonabad University of Medical Sciences, Gonabad, Iran
\end{abstract}

\begin{abstract}
Aims: Endotracheal intubation can increase sympathetic reflex activity result in blood pressure increase, arrhythmia and tachycardia. This study aimed to compare the effects of Clonidine premedication and Fentanyl on hemodynamic factors (heart rate, systolic and diastolic blood pressure) in opium-dependent patients under endotracheal intubation.

Materials \& Methods: This single-blind clinical trial was done on 60 opiumdependent patients undergoing elective surgery under general anesthesia with endotracheal intubation in 2013 at operating room of 15 Khordad hospital. The samples were divided randomly into two equal groups receiving Clonidine and Fentanyl. Systolic and diastolic blood pressure and heart rate were compared before, immediately and $5 \mathrm{~min}$ after intubation. Data were analyzed using Independent-T, ANOVA and Pearson correlation coefficient tests by SPSS 16 Software.

Findings: The mean heart rate, systolic and diastolic blood pressure before endotracheal intubation in both groups was not statistically different $(p>0.05)$ but the mean heart rate, systolic and diastolic blood pressure, immediately after intubation in both groups was maximum and showed significant difference statistically $(\mathrm{p}<0.001)$. Systolic and diastolic blood pressure in Clonidine group, $5 \mathrm{~min}$ after intubation significantly decreased compared with prior to intubation and showed significant difference to the Fentanyl group statistically $(\mathrm{p}<0.001)$.

Conclusion: Using Fentanyl as a premedication before laryngoscopy and endotracheal intubation is less effective than Clonidine to control of hemodynamic parameters in opium-dependent patients.
\end{abstract}

\section{Keywords}

Laryngoscopy [http://www.ncbi.nlm.nih.gov/mesh/68007828];

Intubation [http://www.ncbi.nlm.nih.gov/mesh/68007440];

Heart Rate [http://www.ncbi.nlm.nih.gov/mesh/68006339];

Blood Pressure [http://www.ncbi.nlm.nih.gov/mesh/68001794]

*Corresponding Author

Tel: +985157231113

Fax: +985157231116

Address: No. 10, Mahdi 3, Sa'di Town, Gonabad, Iran

alirezatalai@yahoo.com

Received: September 10, 2014 Accepted: December 24, 2014 ePublished: February 19, 2015 
تاريخ دريافت: 9/19./\%وسا

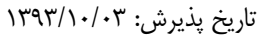

"نويسنده مسئول: alirezatalai@yahoo.com

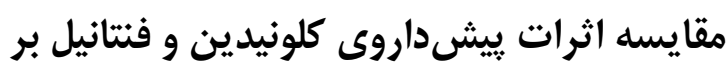

شاخصهاى هموديناميك در بيماران وابسته به مواد مخدر تحت لولهَّذارى داخل تراشه

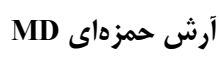

كَروه هوشبرى و اتاق عمل، دانشكده يِيرايزشكى، دانشخاه علوم يزشكى گناباد،

كناباد، ايران

MSc مهدى بصيرىمقدم

كروه برستارى، دانشكده برستارى و مامايى، دانشكاه علوم يزشكى گَناباد، كناباد،

ايران

على محمديور PhD

كروه آموزش يرستارى داخلى و و جراحى، دانشكده يرستارى و مامايى، دانشكاه علوم يزشكى كناباد، گناباد، ايران داني

MSc "علير ضا طلايیى

كروه يرستارى، دانشكده يرستارى و مامايى، دانشكاه علوم يزشكى كناباد، كناباد،

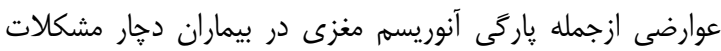

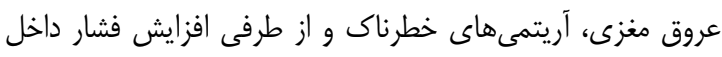

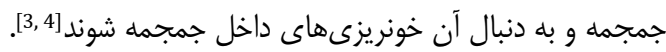

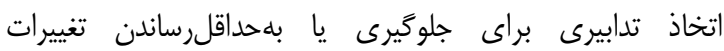

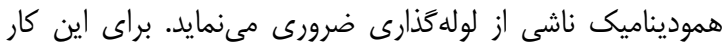

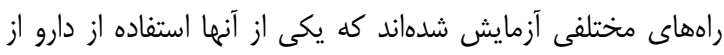
جمله اكونيستهاى كيرندهاى

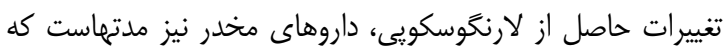

$$
\text { مورد استفاده هستند [6]. }
$$

مطالعات مختلف نشان داده است كه فنتانيل (داروى مخدر صناد منتى

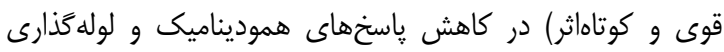

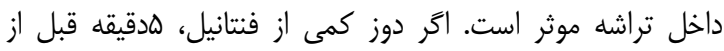

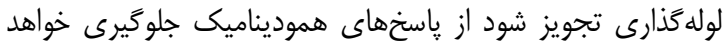

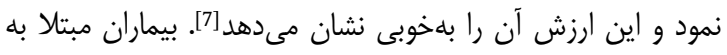

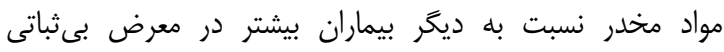

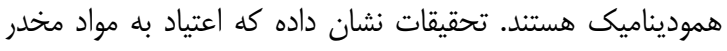

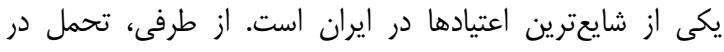

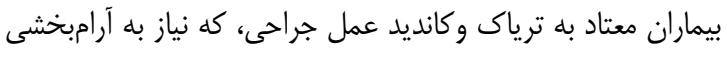

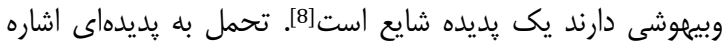

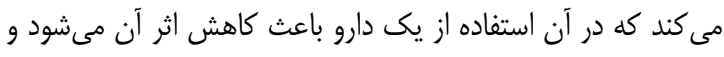

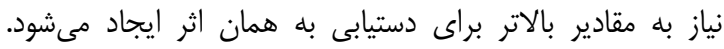

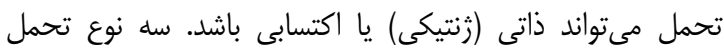

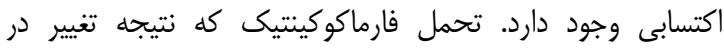

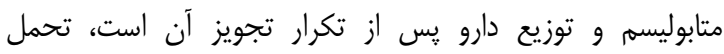

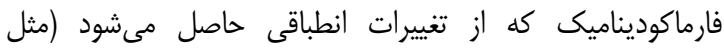

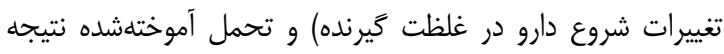
مكانيسمهاى جبرانى است [9].
جكيده

اهداف: لولهَذارى داخل تراشه موجب افزايش در فعاليت بازتابى

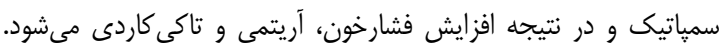

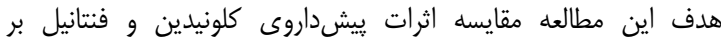
شاخصهاى هموديناميك (ضربان قلب، فشارخون سيستولى و دياستولى)

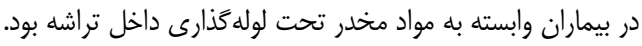
مواد و روشها: مطالعه حاضر كارآزمايى بالينى يكسويه كورى است

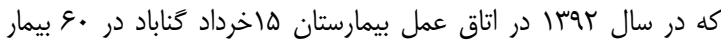

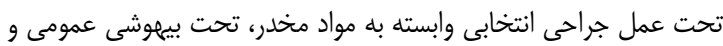

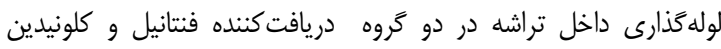

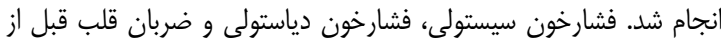

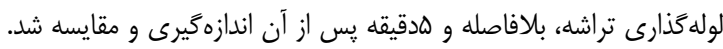

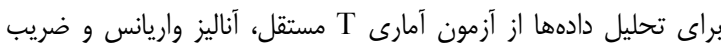

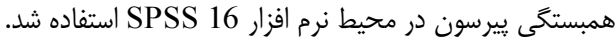

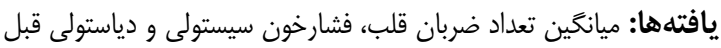

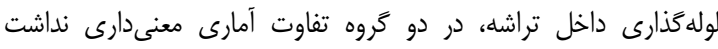

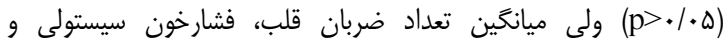

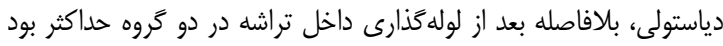

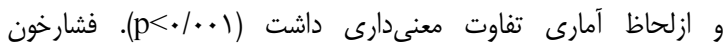

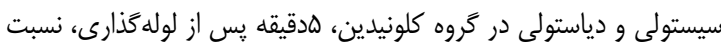

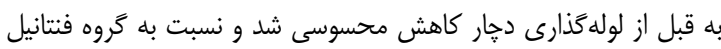

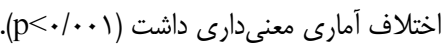

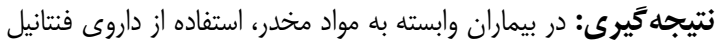

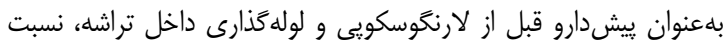

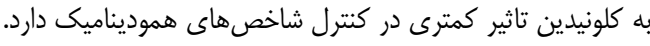

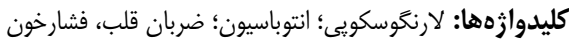




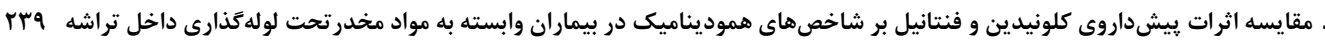

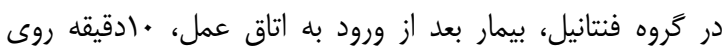

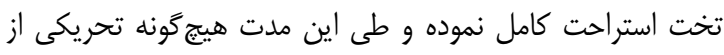

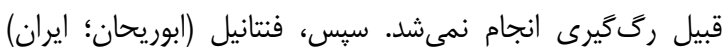

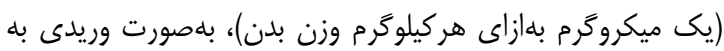
بيمار تزريق مىشد. مدقيقه بعد فشارخون سيستولى و دياستولى و

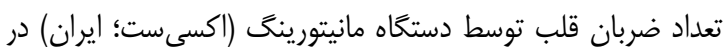

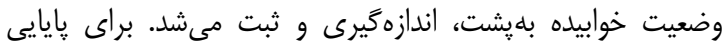

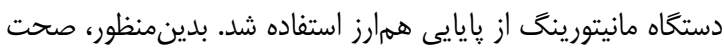

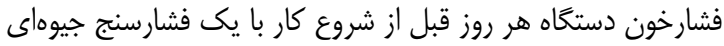

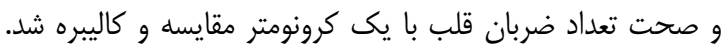

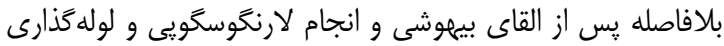

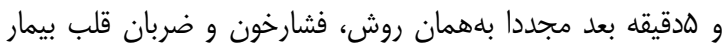

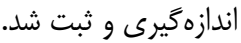

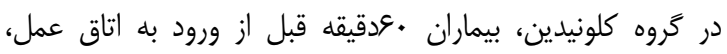

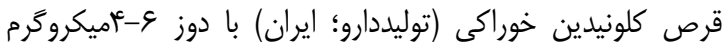

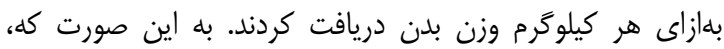

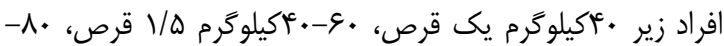

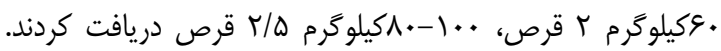

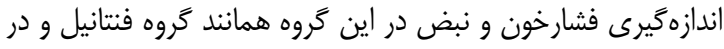

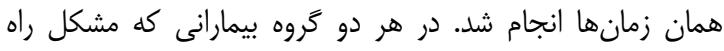

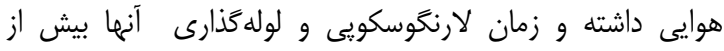

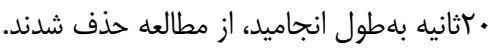
نوع بيهوشى و ساير داروهاى مورد استفاده در هر دو كروه يكسان

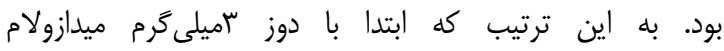

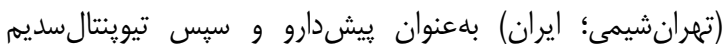

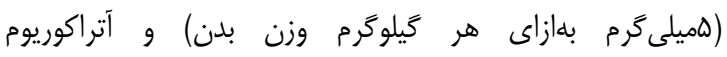

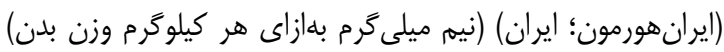

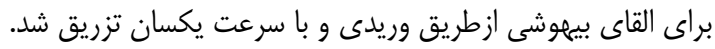

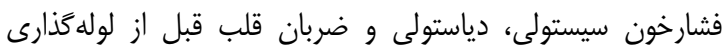

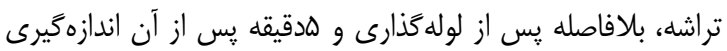

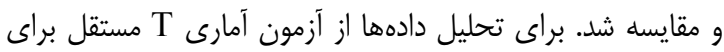

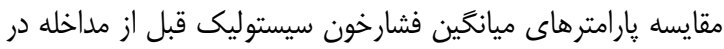

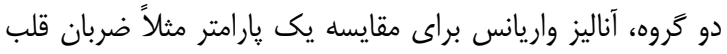

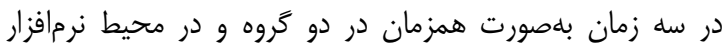
SPSS 16

\section{يافتهها}

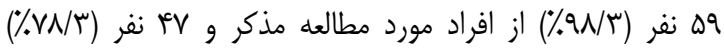

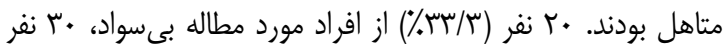

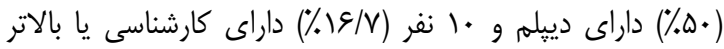

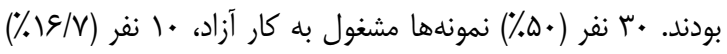

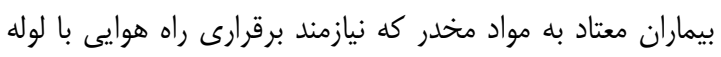
تراشه هستند، با توجه به احتمال ايجاد تحمل نسبت به به مواد مخدر

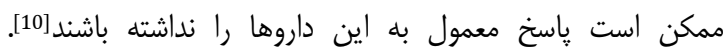

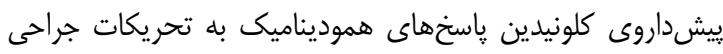

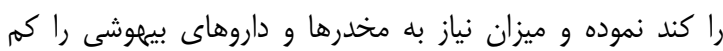

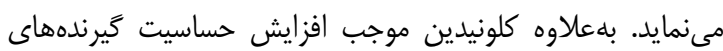

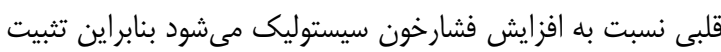

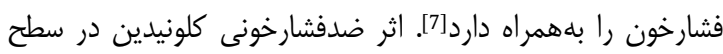

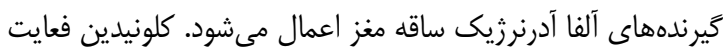

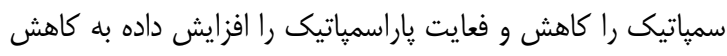

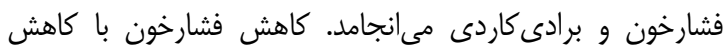

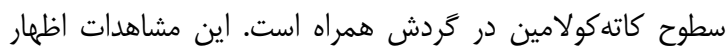

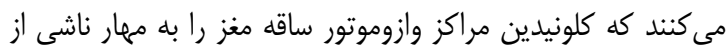

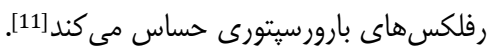

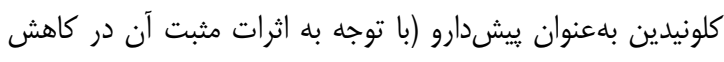

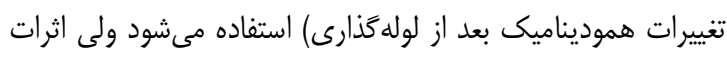

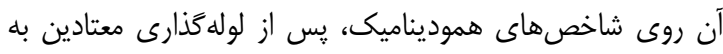

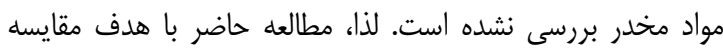

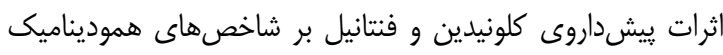

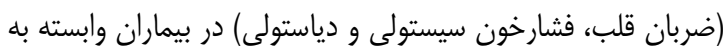
مواد مخدر تحت لوله كذارى داخل تراشه انجام شد.

\section{مواد و روشها}

مطالعه حاضر يك كارآزمايى بالينى يكسويه كورا كور است كه در سال

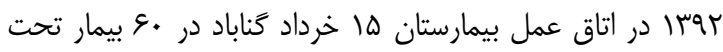

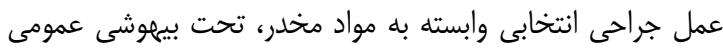

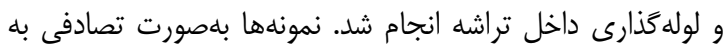

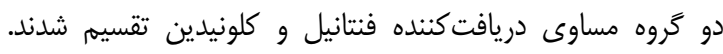

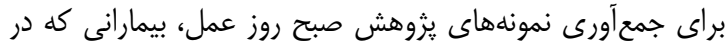

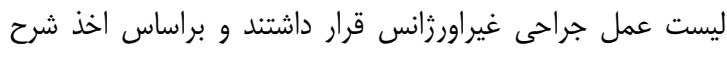
حال توسط فرد آموزشديده به مخدر خوراكى يا يا استنشاقى وابسته

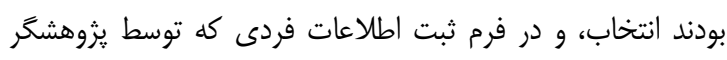

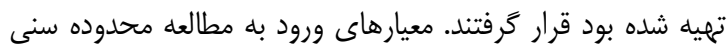

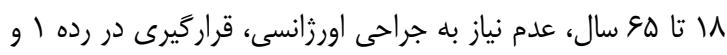

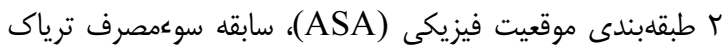

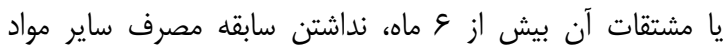

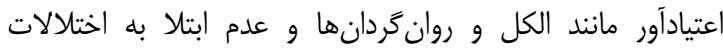

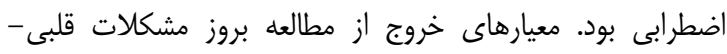

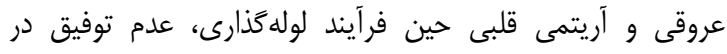

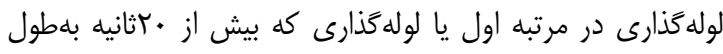

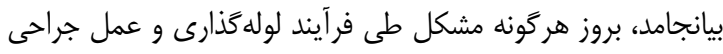

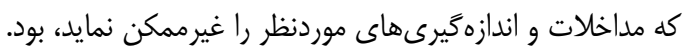




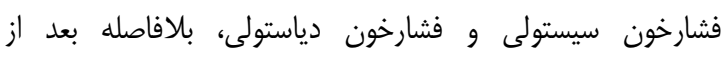

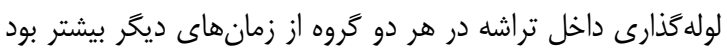

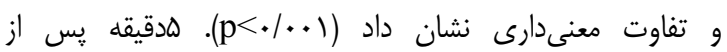

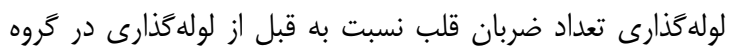

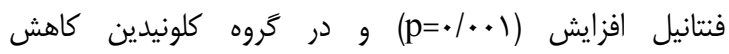

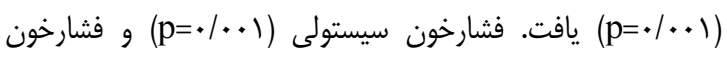

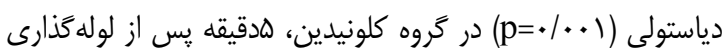

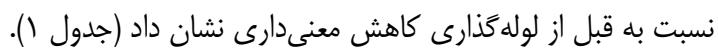

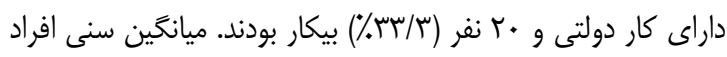

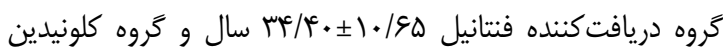
فنتانيل

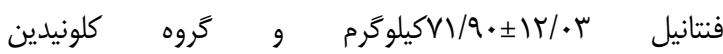

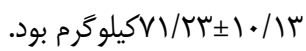

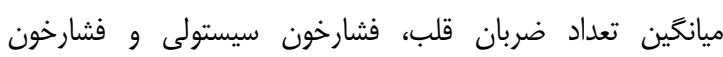

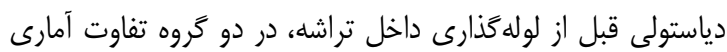

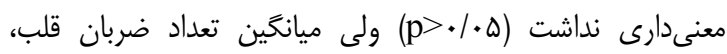

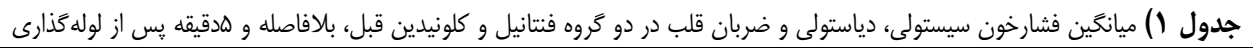

\begin{tabular}{|c|c|c|c|}
\hline هدقيقه يس از لوله كذارى & بلافاصله بعد از لولهَذارى & قبل از لولهَذارى & متغيرها \\
\hline \multirow{3}{*}{$\begin{array}{l}q) / r \Psi \pm I N / r q \\
V \xi / r Y \pm I r / \Delta q\end{array}$} & & & ضربان قلب (عدد) \\
\hline & $1 \cdot \Delta / \Lambda \cdot \pm|\varepsilon / 4|$ & $\Lambda 1 / \Lambda \cdot \pm|f / \Delta|$ & كروه فنتانيل \\
\hline & $\Lambda \Delta / V \Psi \pm|r / \Delta|$ & $v \varepsilon / \Lambda \cdot \pm 1 \cdot / r r$ & كروه كلونيدين \\
\hline \multicolumn{4}{|r|}{ فشارخون سيستولى (ميلىمتر جيوه) } \\
\hline$I T \Delta / \Lambda T_{ \pm} I V / Q T$ & $\mid \varepsilon T / \& \cdot \pm 1 \varepsilon / F \Delta$ & $|r F / r \pm I| / r V$ & كروه فنتانيل \\
\hline $1.9 / r \pi \pm 1 / / r$. & $\mid r N / r T \pm I V / T Q$ & $|r \cdot / r \pm \pm| f / \cdot q$ & كروه كلونيدين \\
\hline \multicolumn{4}{|r|}{ فشارخون دياستولى (ميلىمتر جيوه) } \\
\hline$\lambda \cdot / r \cdot \pm|r / 9|$ & $1.8 / 4 r \pm 11 / \Delta q$ & $V N / \& V \pm N / A T$ & كروه فنتانيل \\
\hline $9 q / \mu \cdot \pm 1 r / \Lambda \varepsilon$ & $\Lambda e / \Lambda \cdot \pm I r / t r$ & $V E / r \mu \pm 11 / \Delta s$ & كروه كلونيدين \\
\hline
\end{tabular}

همسويى دارد. همجنين نتايج با مطالعه شيربمن و همكاران [12] كه

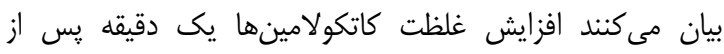

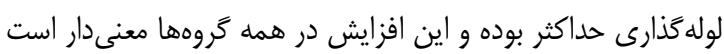
نيز همخوانى دارد. در اين مطالعه، در كروه فنتانيل، فشارخون سيستولى بلافاصله بعد از

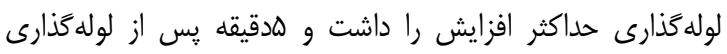

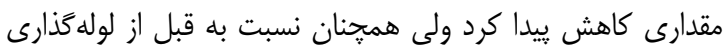

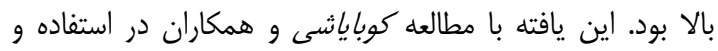

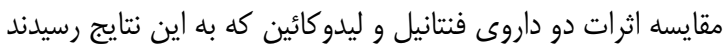

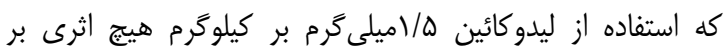

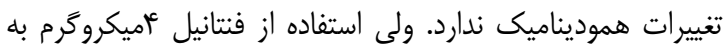

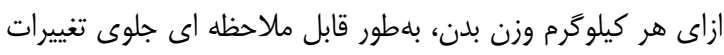

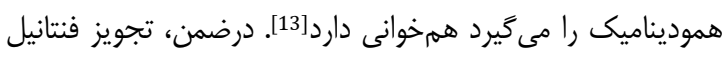

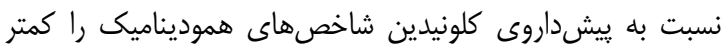

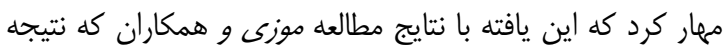

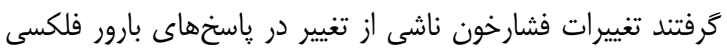

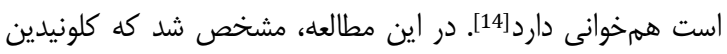

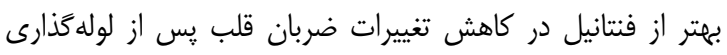

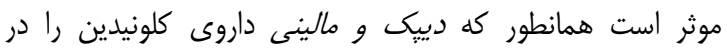

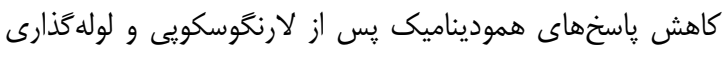

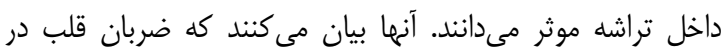

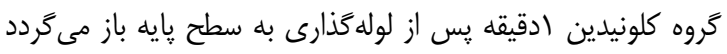

\section{بحث}

در اين مطالعه، در دو گروه تفاوت آمارى معنىدارى از نظر سن

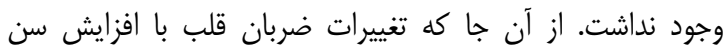

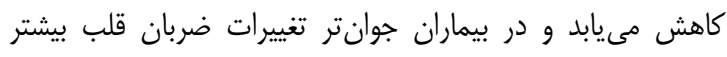

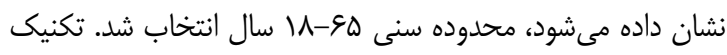

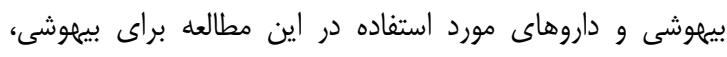

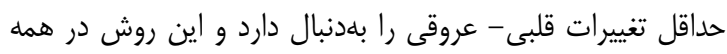
نمونهها باصورت يكسان اجرا شدير

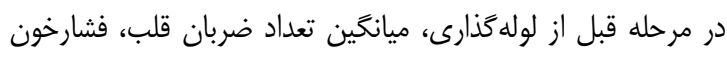

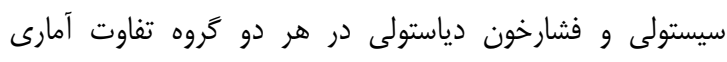

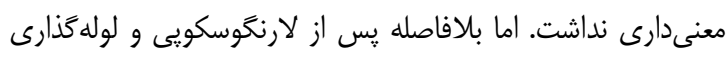

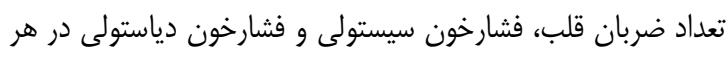

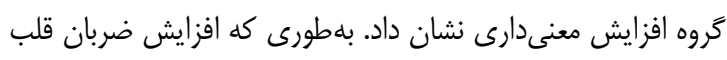

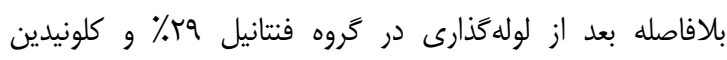

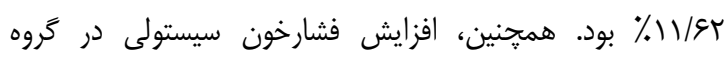

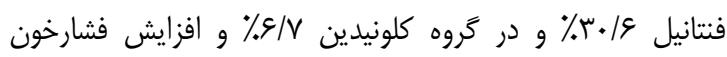

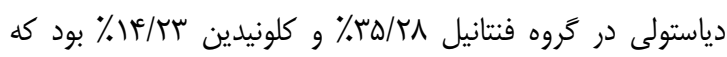

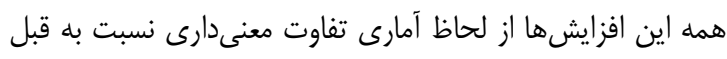

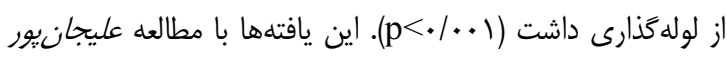

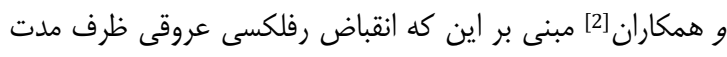

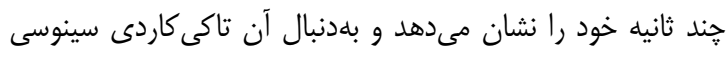
طى זدقيقه به اوج مىرسد و هدقيقه هم بلهطول مى نىانجامد، 


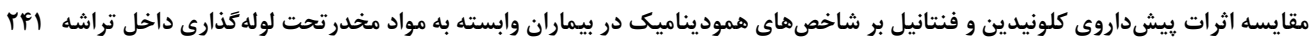

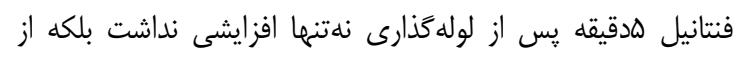

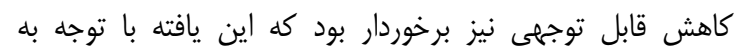

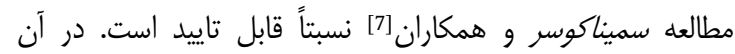

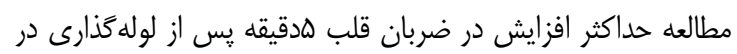

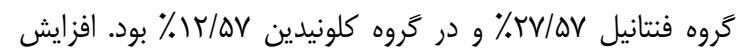

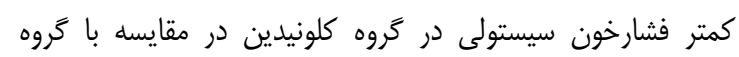

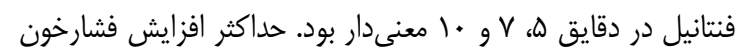

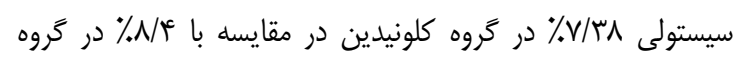

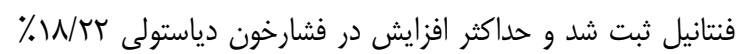

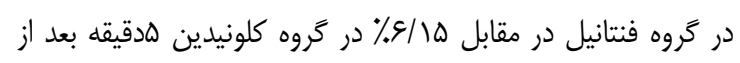

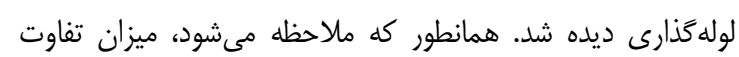

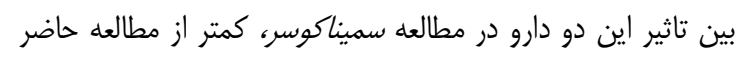

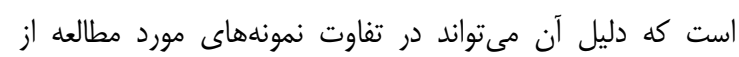

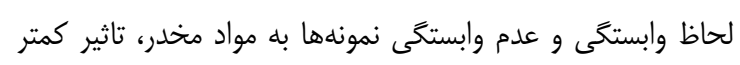

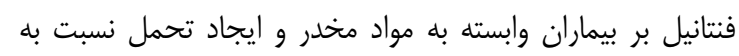
مواد مخدر باشد. از محدوديتهاى اين مطالعه مىتوان به دسترسى سخت بـ به دمان

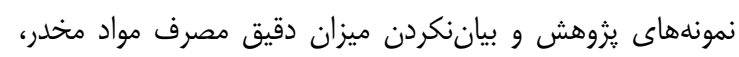

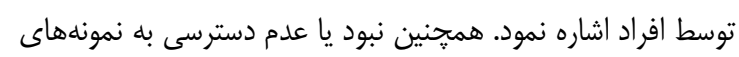

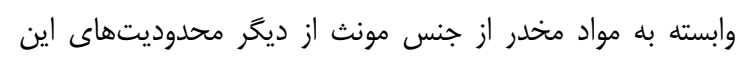

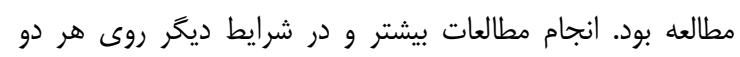
جنس با جامعه آمارى وسيعتر يِيشنهاد مى مطود.

\section{نتيجه كَيرى}

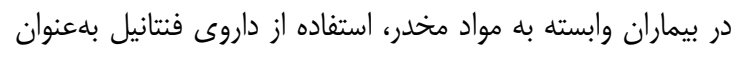

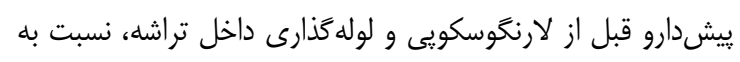
كلونيدين تاثير كمترى در كنترل شاخص هاى هموديناميك دارد.

تشكر و قدردانى: بدين وسيله از كاركنان، مسئولان و يزشكان

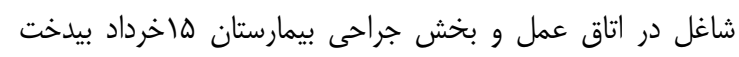

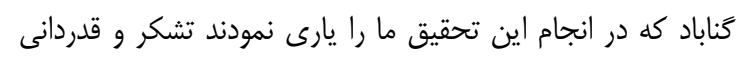

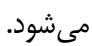

تاييديه اخلاقى: تاييديه اخلاقى اين بزوهش از كميته اخلاق

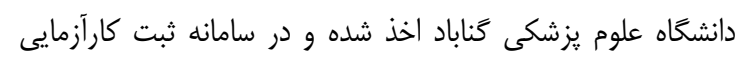

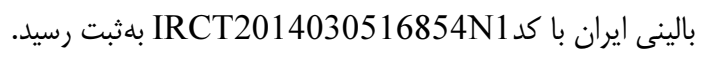
تعارض منافع: موردى توسط نويسندكان گزارش نشده است.

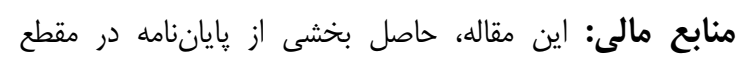

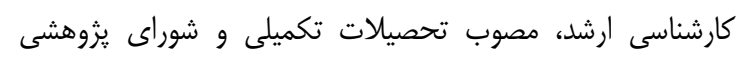

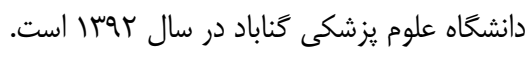

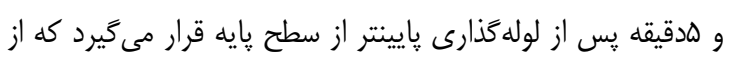

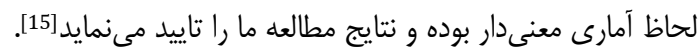

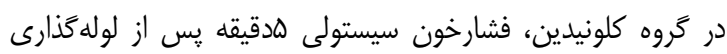

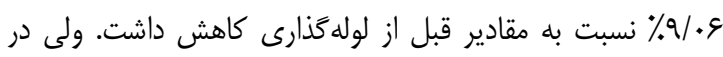

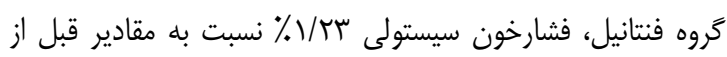

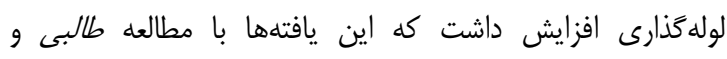

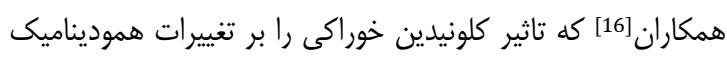

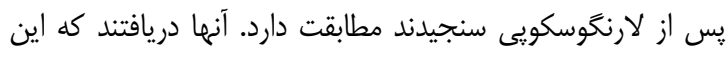

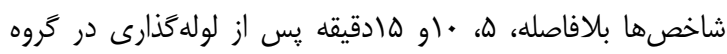

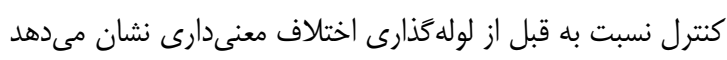

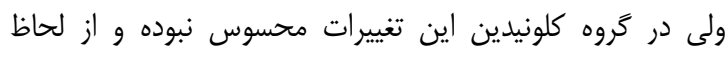

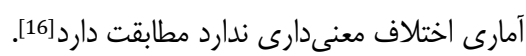

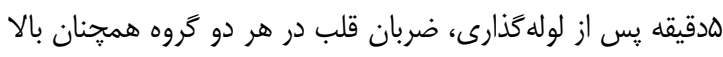

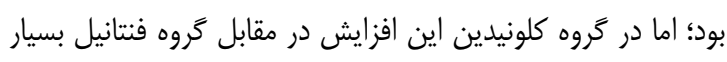

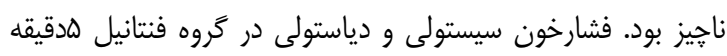

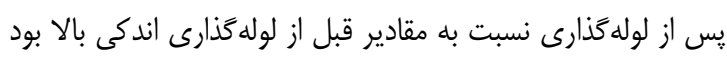

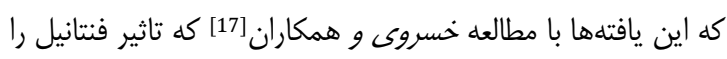

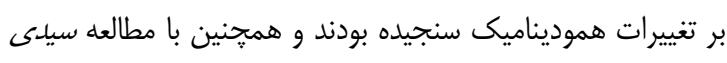

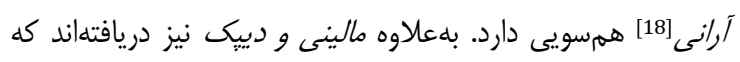

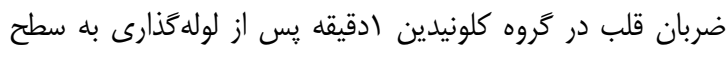

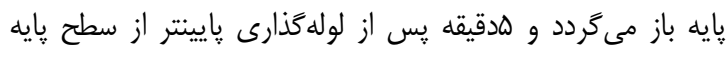
قرار مى گيرد و با نتايج مطالعه ما همخوانى دارد [15.].

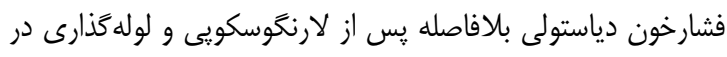

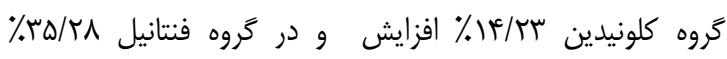

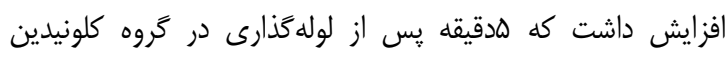

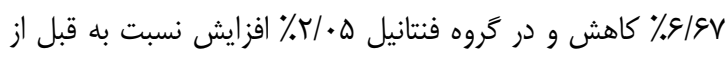

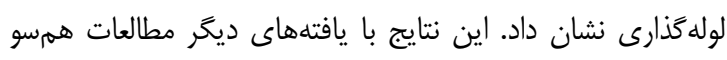
است]

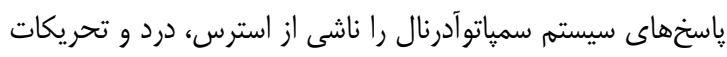

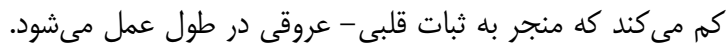

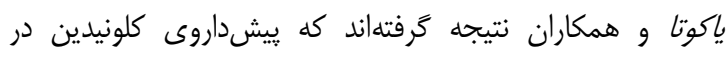

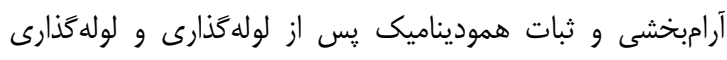

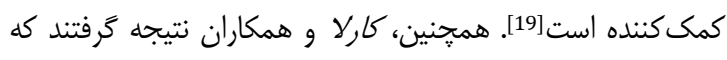

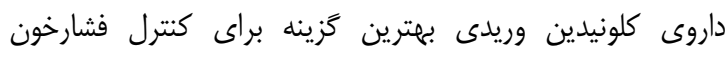

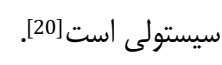
بهطور كلى در اين مطالعه مشخص شد كه افزايش قابل توجهى در

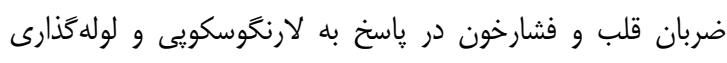

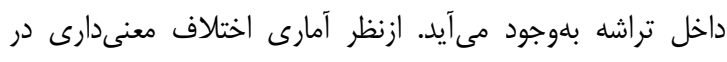

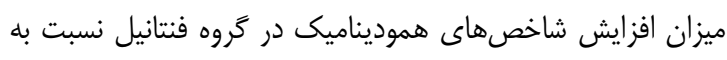

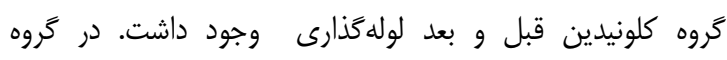

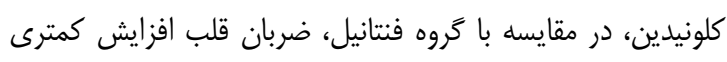

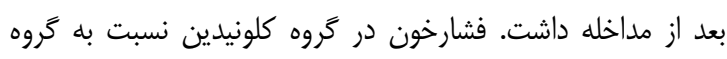


Livingstoon. 2010;27:214.

11- Katzung B, Masters S, Trevor A. Basic \& Clinical pharmacology. New York: McGraw-Hill Medical; 2012.

12- Shribman AJ, Smith G, Achola KJ. Cardiovascular and catecholamine responses to laryngoscopy with and without tracheal intubation. Br J Anaesth. 1987;59(3):295-9.

13- Kobayashi TL, Watanabe K, Lto T. Lack of effect of i.v. lidocaine on cardiovascular responses to laryngoscopy and intubation. Masui. 1995;44(4):579-82.

14- Muzi M, Goff DR, Kampine JP, Roerig DL, Ebert TJ. Clonidine reduces sympathetic activity but maintains baroreflex responses in normotensive humans. Anesthesiology. 1992;77(5):864-71.

15- Dipak LR, Malini KM. Oral clonidine pre medication for attenuation of haemodynamic response to laryngoscopy and intubation. Indian JAnaesth. 2002;46(2):124-9.

16- Talebi H, Nourozi A, Fateh S, Mohammadzadeh A, Eghtesadi-Araghi P, Jabbari S, Kalantarian M. Effects of oral clonidine premedication on haemodynamic response to laryngoscopy and tracheal intubation: A clinical trial. Pak J Biol Sci. 2010;13(23):1146-50.

17- Khosravi M, Azemate S, Sheibani N. Comparison of magnesium sulfate with fentanyl and lidocaine on changes induced by laryngoscopy and intubation during coronary artery surgery. J Med Faculty Gilan Univ Med Sci. 2004;8(52):41-7.

18- Sayyedy Arany H. Effect of intravenous fentanyl on heart rate and rhythm changes induced by tracheal intubation in patients undergoing elective abdominal surgery. Hormozgan Med J. 2001;6(2):39-45.

19- Yokota S, Komatsu T, Yano K, Taki K, Shimada Y. Effect of oral clonidine premedication on hemodynamic response during sedated nasal fiberoptic intubation. Nagoya J Med Sci. 1998;61(1-2):47-52.

20- Kalra NK, Verma A, Agarwal A, Pandey HD. Comparative study of intravenously administered clonidine and magnesium sulfate on hemodynamic responses during laparoscopic cholecystectomy. J Anaesthesiol Clin Pharmacol. 2011;27(3):344-8

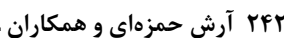

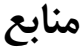

1- Tabari M, Alipour M, Ahmadi M. Hemodynamic change occuring tracheal intubation by direct laryngoscopy compard with intubating laryngeal mask airway in adult: A randomized comparison study. Egypt $\mathrm{J}$ Anaesth. 2013;29(2):103-7.

2-_Alijanpour E, Amry P, Rezaei Moghaddam A. Comparison of intravenous magnesium sulfate and lidocaine on hemodynamic changes during intubation. J Babol Univ Med Sci. 2006;8(4):20-5.

3- Ko DD, Kang H, Yang SY, Shin HY, Baek CW, Jung $\mathrm{YH}$, et al. A comparison of hemodynamic change after endotracheaql intubation by the Optiscope and the conventional laryngoscope. Korean J Anesthesiol. 2012;63(2):130-5.

4- Kovac AL. Controlling the hemodynamic respose to laryngoscopy and endotracheal intubation. J Clin Anesth. 1996;8(1):63-79.

5- Brunton L, Chabner B, Knollman B. Goodman and Gilman's The Pharmacological Basis of Therapeutics. $12^{\text {th }}$ ed. New York: McGraw-Hill Professional; 2001. 6- Ebert JP, Pearson JD, Gelman S, Harris C, Bradley EL. Circulatory response to laryngoscopy. The comparative effects of placebo. Can J Anaesth. 1989;36(3 Pt 1):301-6.

7- Sameenakousar, Mahesh, Srinivasan KV. Comparison of fentanyl and clonidine for attenuation of the haemodynamic response to laryngocopy and endotracheal intubation. J Clin Diagn Res. 2013;7(1):106-11

8- Jabbary Moghaddam M, Ommi D, Mirkheshti A, Dabbagh A, Memary E, Sadeghi A, et al. Effect of clonidine premedication Upon postoperative shivering and Recovery Time in patients With and Without Opium Addiction After Elective leg Fracture Surgeries. Anesth Pain. 2013;2(3):107. 10.

9- Imani F. Text book of pain. J Anesthesiology. 2013;3(3):0-133.

10- Miler RD, Eriksson LI, Fleisher L, Wiener-Kronish JP, Young WL. Miller's Anesthesia. $7^{\text {th }}$ ed. London: Churchil 